\title{
SYSTEMIC REFLEXIVITY IN RESIDENTIAL CHILD CARE: A PEDAGOGICAL FRAME TO EMPOWER PROFESSIONAL COMPETENCE
}

\section{Laura Formenti and Alessandra Rigamonti}

\begin{abstract}
This position paper offers a pedagogical frame to empower professional work in residential child care. Jobs in this demanding field are characterized by daily relationships with children of different ages, needs, and cultural backgrounds. There is a need for effective communication and interaction with them, their families, co-workers, other professionals, and care agencies, as well as with the larger community. This complexity brings uncertainty and the necessity of thinking and acting in a sensitive way in order to open possibilities for systemic transformation at the micro, meso, and macro levels. In this framework, we focus on reflexivity as a meta-competence - a set of specific postures, competences, and attitudes that characterize expert professional action. A thorough literature review on reflexivity in social work and child protection is aimed at clarifying the meanings, uses, and features of this concept. We claim that systemic reflexivity can be used as a framework, a methodology, and a set of tools to empower professional work by enhancing emotional, cognitive, and epistemic self-awareness, systemic wisdom, abduction, and active listening. To help workers and teams develop these five competences, a self-directed learning module is currently being designed, based on systemic and narrative perspectives, and transformative learning theory.
\end{abstract}

Keywords: residential child care, reflexivity, competence, systemic approach, narratives

Laura Formenti PhD (corresponding author) is a Professor of General and Social Pedagogy at Milano Bicocca University, Piazza Ateneo Nuovo, n. 1, 20126 Milano, Italy. Email:

laura.formenti@unimib.it

Alessandra Rigamonti PhD is a Research Fellow at Milano Bicocca University, Piazza Ateneo Nuovo, n. 1, 20126 Milano, Italy. Email: alessandra.rigamonti@unimib.it 


\section{Working in Residential Child Care: The Need for a Systemic Pedagogical Frame}

In this paper, we reflect on training educational care workers with a view to creating a basis for designing a solid curriculum aimed at empowering workers, and building a stronger professional identity based on competences. This study is part of a larger project ${ }^{1}$ about residential child care (RCC), where our task is to develop the pedagogical frame for a massive open online course (MOOC) to be offered to workers and agencies to provide self-directed learning both in the workplace and during the passage from university to work. We are persuaded that, in order to guarantee better outcomes in RCC, we must empower workers by offering, besides solid knowledge, a competence-based learning context, where they can develop their reflexivity.

Our questions are: What do professionals in RCC need in order to become more effective? Which competences are entailed by good, or "good enough", practice? When is a professional considered to be, and able to recognize herself or himself as, "competent"?

Researchers, professional associations, and experts have developed principles, requirements, and standards about child protection and care, and emphasized the need for trained and experienced workers endowed with complex relational capacities (Garfat, 2013; Gharabaghi, 2020; Mattingly et al., 2012). It is by and large recognized that care workers play a critical role in RCC outcomes (Knorth et al., 2010). In many countries, they need a specific qualification at entry level, but there is little standardization as to how the needed competences are defined, assessed, developed, and learnt in the workplace.

Although specific requirements depend on the country's welfare policies and dominant cultural models, there is a push towards professionalism. For example, in Italy, since 2017 all these workers must have a Bachelor of Arts in Educational Sciences; they are named "professional educators", while in other countries workers may be social workers or nurses, or assistants having either no specific qualification or a generic degree. Professionalization can be described as a composition of different dimensions: awareness of role, professional identity, juridical framework, commitment to excellence, and competence. The latter is our focus here, but all dimensions interact in entangled ways. For example: a worker may be recognized as competent by colleagues and clients, but a weak juridical understanding and low status could interfere with his or her agency, a component of competence. Increasing bureaucratization, on one side, and academic training, on the other, can undermine or undervalue relational competences in favor of other attitudes. Professionalization can thus create manifold problems having complex dynamics.

Professional empowerment entails being recognized as a competent practitioner, as well as feeling and acting like one; it comprises cultural, relational, and communication skills, but skill is

\footnotetext{
1 "Empowering Residential Child Care through Interprofessional Training", funded by the European Union's Erasmus Plus program. The principal investigator is Eeva Timonen-Kallio. For more information, see https://ercci.turkuamk.fi/en/
} 
not synonymous with competence, which is more complex, context-based, and transferrable. Timonen-Kallio and Hämäläinen (2019) define six areas of competence informed by a social pedagogy framework: educating for community; promoting participation in daily life; planning and maintaining the child's social integration process; implementing specific and intentional educational interventions; collaborating in internal and external multiprofessional teams; and continuing professional learning and education. These authors value relationships, the quality of daily life in a homelike context, and collaboration between different professionals, all of them orientated to children's well-being and participation. Other authors highlight the need for knowledge based on solid evidence (Akin et al., 2016; del Valle et al., 2015; James, 2017). These differences evoke paradigms and hidden pedagogical models. The traditional model in child care is based on a strongly relational profile, too often downplayed as "the good-hearted type", but the push towards specialization and evidence-based practice exhorts practitioners to be objective, and base their decisions on the application of models and rules derived from research. This binary divide is mirrored in training: on one side, it is aimed at developing life skills such as communication, listening, sympathy, responsiveness, and emotional self-awareness; on the other side, it seeks to enforce the application of specific techniques and acknowledged models. The dichotomy is not helpful: too often, it creates a clash of paradigms in the community of child protection, especially among professionals with different curricula and experience.

We consider RCC a complex system, needing both rational and relational answers, different kinds of research, and a strong connection between theory and practice. It also has a strong commitment to social justice: this means that ethical and political issues cannot be eliminated from the framework. Reflexivity is a way to connect different forms and levels of knowledge within a larger framework, so it can be helpful in overcoming the opposition between the "good old" relational practitioner and the "new" skilled and trained one.

Critical thinking is an essential part of reflexivity. Care work entails certain inherent contradictions; for example, Harder et al. (2017) signalled the need to balance rules and freedom. Our research with care leavers (Formenti et al., 2020) shows that a competent social worker must embody layered and contradictory dimensions. The influence of personal assumptions and values, cultural biases, and power issues is strong. How do workers in RCC learn to connect their knowledge, skills, and abilities, their motivations and values, with the capacity to act in ways that are useful and effective, and consistent with emerging needs and demands, while keeping in view a possible transformation towards the good? Competence can only be recognized when it is enacted in a real situation and its outcome is satisfying for different actors and from different perspectives.

We therefore focus our attention on reflexivity as a meta-competence and a tool for professional growth. We search for differences between reflection and reflexivity, and for criteria to define and operationalize reflexivity in a systemic framework. We connect reflexivity to the learning potential of narratives and use transformative learning (Formenti \& West, 2018; Mezirow, 1991) as a basis for training design. Our final aim is to suggest that systemic reflexivity can be 
International Journal of Child, Youth and Family Studies (2020) 11(4.2): 115-139

supported by exercises, using stories and questions that help practitioners to develop their awareness, relationality, responsibility, and agency.

\section{Literature Review: Reflexivity and Reflection in Child Protection and Social Work}

Reflection is the capacity to think about an action and its conditions, and to select an action conducive to desired outcomes; when we reflect, we ponder what we know (i.e., conscious information) from both external sources and introspection, and we make judgements that may eventually guide our actions. Reflection is difficult and does not come automatically: it is a construction, an act of interpretation that entails some practice. Schön's (1983) seminal work established reflection as relevant to professionals in general, and gave rise to an extensive literature exploring the concept.

Reflexivity, as a concept, is far less clear. The word is sometimes used as a synonym of reflection, but may also be introduced to signal another meaning. For example, one of us offered a view of reflexivity in the area of adult learning and education (Formenti, 2017; Formenti \& West, 2018) as a more-than-cognitive competence, entailing memory, perception, interaction, emotion, imagination, and the use of presentational languages. It includes unconscious processes and ongoing interaction with a material and human context.

If the need for reflection in social work practice now seems universally accepted (Ferguson, 2018), the need for - or even a definition of — reflexivity is less widely shared. Despite the presence of this term in social work literature since the 1990s, it is often used without a careful and critical effort to define it. In doing this literature review, our questions are: What does reflexivity mean? Which presuppositions and perspectives of meaning, which criteria and dimensions of reflexivity, are defined in the literature? How can they enhance social work, educational care, and child protection?

Since our aim is to highlight the role of reflexivity in training RCC workers, this review is mainly based on papers that use "reflexivity" and "reflection" in relation to practice in child protection and care. We set aside other contexts, for example, research — where reflexivity has gained prominence after the turn to interpretivism (Alvesson \& Sköldberg, 2000; Maton, 2003; Simon \& Chard, 2014), but we did look into family therapy, for its influence on social work (Bingle \& Middleton, 2019; Partridge et al., 2019; Watson, 2019).

Reflexivity is referenced in papers about theory, research, practice, or training in social work, management, psychological intervention, and supervision, whereas reflection is generally referenced in relation to the improvement of professional practice, often within a frame of social justice and emancipation. In their literature review, D'Cruz et al. (2007) chronicled multiple meanings for "reflexivity", "reflectivity", "critical reflection", and "reflection"; they deplored the lack of clarity and interchangeable use of these terms. They described three "variations" of meaning: (1) the ability of social workers, via an intrapsychic process, to acquire information and 
make choices based on knowledge in a given relational situation; (2) critical and self-critical approaches to practice, questioning the neutrality of knowledge and recognizing the role of power in the relationship; and (3) self-awareness and practitioners' active use of their emotional responses — instead of repressing or controlling them — and their position in the relational process.

By questioning the relationship between knowledge and practice, reflexivity introduces the theme of uncertainty. In the first variation above, the uncertainty arises from the complexity of life in contemporary society; in the second and third variations, it is due to awareness of the situated construction of knowledge that characterizes practice and makes any account a partial one. We acknowledge the risk of fuelling relativism (D'Cruz et al., 2007), but we will show how a systemic, relational, and situated approach to reflexivity can help one avoid adopting an "anything goes" position.

Some authors offer specific terms, whose meaning is peculiar to their approach. For instance, Kondrat (1999) uses "critical reflective" to characterize social work that is connected to and aware of the sociohistorical context. Fook frames "reflectivity" (2002) and "critical reflection" (2012) as processes that turn the experience of practice into learning; she suggests (2002) that "reflection/reflectivity" comes from case management and the need to improve practice, while "reflexivity" comes from qualitative and ethnographic research and the need to locate the observer in the picture.

In our analysis, we used systemic and constructivist lenses to highlight different layers of construction and interpretation of reflexivity, based on the micro, meso, and macro levels of systemic functioning (Formenti \& West, 2018):

- The microlevel focuses on the individual as a system. Here, reflexivity is constructed as a process connecting emotional, cognitive, physical, biographical, and situational aspects in the individual social worker's experience.

- The mesolevel focuses on interaction and the circularity of human communication, especially in systems with a history of relationships. Reflexivity is co-constructed by communication and conversations within a social organisation and collective mind - a family, team, or group. These systems tend to stabilize in time and develop their own scripts, myths, rules, and identities, along with a sense of belonging ("us"). An RCC unit can be regarded as such a system.

- The macrolevel refers to the larger context: cultural, social, economic, political, and legal factors impinge on social work. Here, reflexivity relates to the awareness of the role of social structures, discourse, dominant perspectives, and societal myths in shaping practice.

In addition to this analysis, we identified two main topics: the influence of the systemic approach in shaping reflexive and reflective practices and the relationship between reflexivity and narrativity. 
International Journal of Child, Youth and Family Studies (2020) 11(4.2): 115-139

\section{Self-Reflexivity as a Feature of the Microlevel}

Many authors have addressed the need to build awareness of how practice is shaped by each professional's unconsciously held assumptions, as well as personal ideas and theories. Bingle and Middleton (2019) defined self-reflexivity as a position enabling workers to distance themselves from the dominant language of child protection that pushes them to find "the right answer". Roets et al. (2017) studied the capacity to connect perception, interpretation, and communication in practice, which may be sustained by the creation of stories. For example, when writing a report, it may be more useful to view it as a practice of storytelling, not a bare recitation of facts. Defining and solving a problem entail engaging in an ongoing construction; therefore, an approach involving the blind application of protocols and case management rules is likely to be ineffectual.

Reflexivity creates a frame for bringing attention to everyday practice and developing a capacity to interrogate, present, and defend one's own ideas (Magnuson et al., 2012). Some authors (Andrew, 2015; Taylor \& White, 2001) have questioned the dimension of judgement unavoidable in social work - and its moral and emotional implications: how do we develop our ideas, how do we bridge knowledge and feelings, values and actions, and what are the consequences?

According to Ferguson (2018), the reflective process is triggered by the sensorial, emotional, and experiential impact of social work. For example, anxiety is frequently mentioned by social workers and becomes problematic when they are unable to start an internal supervision Ferguson's term for a process beyond generic reflection — which gives containment to ideas and feelings.

Papp and Rácz (2016) considered self-reflexivity to be a supportive background and protective factor for social workers: without it, they would not be aware of the importance of expressing themselves, taking a position, or making a statement. Along the same lines, Kearns and McArdle (2012) considered reflexivity to be a key feature of resilience, enabling professionals to think in depth and make explicit statements or correct them. The title of their paper — "Doing it right?" alludes to the construction of a problematic (disempowering) narrative that may haunt newly qualified social workers.

\section{Shared Reflexivity as a Feature of the Mesolevel}

Social work is a relational and organizational job, entailing many systems and levels of interaction. Enduring relationships, groups, and organizations constitute systems that self-organize and learn: reflexivity, then, is not only a feature of the individual, but it can be (and needs to be) implemented in the organization of social work so that feedback and circularity of communication can sustain transformation. Many papers make reference to systemic theories and practices (Bingle \& Middleton, 2019; Dugmore et al., 2018; Jude, 2018; Papp \& Rácz, 2016; Partridge et al., 2019; Watson, 2019) that bring to the forefront relationships and contexts rather than individual features. The systemic approach to social work defines problems as relational and social, not intrinsic to the 
person. The constructionist paradigm is used to say that reality is not fixed — not "out there" — but is co-created.

Partridge et al. (2019) considered the reflexive process to be a key systemic principle, a collective action that raises the whole system's capacity to respond to needs by enhancing shared awareness. Dugmore et al. (2018) promoted a model of embedded and embodied systemic supervision that fuels reflexivity, resilience, and a relationship-based practice. This reflexive (rather than prescriptive) practice of supervision is potentially transformative for the team and any others involved. It creates a pause and a space for "safe uncertainty" (Mason, 2015, as cited in Dugmore et al., 2019). The composition of different voices, theoretical perspectives, and domains of action helps the practice overcome the risk of searching for a single truth. Truths are always provisional and open to revision. Lack of certainty may be the first step towards hope.

This framework allows social workers to take an open stance in their ongoing relationships with children, families, colleagues, and other agencies, avoiding the stagnation or stereotyping of programs and the disempowering posture of "zombie social work" (Forrester, 2016).

Feedback and circularity characterize social work practice: the effectiveness of an intervention or program is collectively evaluated by the whole staff using a variety of sources. Group reflective supervision (Bingle \& Middleton, 2019) offers the possibility to exert curiosity and be open to the existence of multiple interpretations of reality - sometimes called "the multiverse", a term we have adopted in this article. Several authors (Bingle \& Middleton, 2019; Dugmore et al., 2018; Partridge et al., 2019) have associated reflexivity with the professional stance of "curiosity", a concept proposed by Cecchin (1987) as an advancement of systemic "hypothesizing" (Selvini et al., 1980). The latter, initially introduced as a guideline for conducting family therapy interviews, is suitable for rethinking social work intervention as based on the multiplication and interconnection of different ideas. It introduces a second-order perspective that amends linearity and welcomes multiple truths, calling for respect and recognition of the value differences in the collective mind (family and team). Curiosity facilitates teamwork in the consideration of all the complex relational and contextual factors entering the evaluation of a situation and in taking shared decisions about the intervention. It is related to "being in relationship" (and not only doing) with other professionals and clients. It sustains the development of new ideas and possibilities in intervention; for example, involving the family in the reflexive process hence allowing the cocreation of new directions instead of blaming, patronizing, or giving instructions to parents.

A relevant issue in social work is power, both in relationships with clients and among professionals. When professionals work towards emancipation, participation, and voice in child protection, shared reflexivity questions the impact of power, without forgetting the ultimate authority of statutory and legal aspects in the context of intervention. The very notion of "child at risk" raises issues of power as a discursive construct shaping practice (Roets et al., 2017). The complexity of dealing with child protection while enhancing parents' competence frequently gives rise to dilemmas in social work. 
International Journal of Child, Youth and Family Studies (2020) 11(4.2): 115-139

Watson (2019) addressed the issue of keeping an ethical and effective position towards children and towards parents, who might feel forced to follow instructions because of the threat of child removal if they do not comply. Reflexivity allows the sharing of authority between therapists (or social workers) and parents. Professionals can support families' reflexivity about parenthood and care. Ferguson (2003) defined the addressees of intervention as potentially "creative reflexive citizens": it is the professionals' task to involve them in their own life-planning, to invite them to disengage from toxic relationships or the psychological inheritance of trauma, and learn how to act critically, to reflect on their actions, and reshape their biographies.

\section{Cultural Reflexivity as a Feature of the Macrolevel}

When reflexivity is enacted, one can hardly avoid considering the larger system and challenging the dominant discourses in social work, whereby expert professionals are expected to wield persuasive, even coercive, power over vulnerable and powerless families or individuals. In 1997, Ferguson had already suggested that institutions and social environments are parts of the reflective process; he criticized reflection as overly individual, conscious, and intentional, but saw reflexivity as entailing the collective, institutional, and social levels.

Reflexivity reveals the influence of structures and discourses - as shaped by the institutional, legal, social, and historical contexts - on social work. Epistemic reflexivity, a cornerstone of Pierre Bourdieu's work (Maton, 2003), highlights the hidden role of dominant knowledge in the professional construction of practice (White, 1997) and helps to overcome some limits of "mere reflection" that arise from the overly individualistic and even narcissistic features of many contemporary reflective practices. Reflexivity needs to be epistemological and collective and show how the relations between knowledge and practice are mediated by social discourse. Any knowledge depends on epistemological conditions, and the latter are related to cultural and social frameworks.

Taylor and White (2001) pointed out that practising reflexivity in social work means to interrogate the construction of interpretation as a feature of any process of description. How was a certain description and interpretation achieved, and which authority was used to claim knowledge or understanding? The social and historical context affects the way practitioners build their knowledge, and act and react to families and children (Taylor \& White, 2001). They are expected to use assumptions from attachment theory, child development theory, biological psychiatry, and established professional models in interpreting children and families' lives and in taking practical decisions towards them. Knowledge, research, and theories that are historically constructed (hence, limited) become pillars of practice, used to understand, interpret, and evaluate the clients' actions and reactions, as well as to justify choices.

Monson (2020) imagined the possibility of creating spaces of reflexivity for professionals to challenge discourses - for example, developmental discourses and dominant family models — in order to build positive attitudes. These spaces should be part of service delivery or constructed informally, but in either case, they need to be supported by the organization. 
International Journal of Child, Youth and Family Studies (2020) 11(4.2): 115-139

\section{Systemic Reflexivity in Social Work Practice}

The systemic approach is not new in the supervision, training, and practices of social work and child protection (Bingle \& Middleton, 2019; Cameron et al., 2016; Goodman \& Trowler, 2012; Munro, 2011; Partridge et al., 2019; Santello et al., 2017; Sebba et al., 2017). This approach, embedded in second-order cybernetics (von Foerster, 2003) and focused on circular dynamic processes of communication and interaction, considers persons, groups, and institutions as interacting dynamic systems (i.e., minds; see Bateson, 1979) following coherent paths of learning, differentiation, and transformation. It is a family-focused approach, based on strengths and giving parents and children the capacity to cope with their situation (Sebba et al., 2017). "Good enough" evolving relationships and an atmosphere of trust, hope, playfulness, and creativity are central to systemic child and family care.

Reflexivity is, then, a key systemic principle (McNamee, 2009; Partridge et al., 2019). Krause (2012) introduced the history of this concept by referring to Gregory Bateson $(1972,1979)$ and his anthropologic work. Bateson questioned his own field observations from the point of view of the observed, concluding that we cannot be sure of our interpretations of another culture, since any explanation depends on the observer's culture and perspective. There is no neutrality or objectivity when it comes to observing the other. However, Bateson did not abandon the idea of understanding this fundamental recursivity (reflexivity) in human knowledge, a "second-order principle" (von Foerster, 2003) that includes the observer in the domain of knowledge construction. Knowledge is mediated by our senses and cultural perspectives, we cannot escape our cultural frameworks when we observe, evaluate, and interpret a situation, an interaction, a family, or a program of intervention. The awareness of our partiality, its roots and consequences, reveals our "posture" or "positioning", illuminating the "what" and "how" in our doing as well as in our feeling, thinking, and reflecting, always in relation to others' feelings and reactions - that is, in a situated context. The process of reflexivity allows the observer — and the observing group, community, or institution - to grasp an opportunity to question the practice and its effects. So, reflexivity is observing observation and reacting to it (Burnham, 2005; von Foerster, 2003).

A specific definition of reflexivity comes from the systemic theory of coordinated management of meaning (CMM), based on the hierarchy of different contexts of communication. Pearce and Cronen (1980) identified six hierarchical levels, beginning with the content of a message, which is defined by its command, which is defined by the episode, and continuing with the relational, biographical, and cultural levels. In the management of meaning, the lower level is framed by the higher, which provides the context in which to interpret it correctly. However, a "reflexive loop" (one that is directed or turned back on itself) happens when two levels are organized in such a way that each of them acts as the context for the other (Cronen et al., 1982). This entanglement adds the complication of recursion, but instead of seeing this as a problem, the authors invite us to recognize this as a constitutive feature of human communication. Recursivity is always present in the process of meaning construction: meaning affects interactions and is recursively (reflexively) affected by them. 
In accordance with this framework, Daniel (2012) proposed that cultural reflexivity, rather than self-reflexivity, should be a feature of therapy: the meeting of different belief systems requires a negotiation of cultural identities, which may result in the creation of new meanings and new ways to engage with otherness and difference. When we interrogate the dominant culture and its way of building otherness, we see ourselves in the eyes of the other and learn to identify some aspects of our habitus that might otherwise remain invisible. All professional interactions are in some way cultural interactions, entailing power and the impact of remote and recent histories of oppression, racism, and marginalization. So, cultural reflexivity can sustain professionals in learning openness, transparency, and reciprocity. In order to communicate across differences, they also need to take risks and leave their comfort zones. Daniel (2012) focused on therapy, but the same principles can be applied to social work. All of us, indeed, should aim for greater sensitivity with regard to how "othering" operates in favor of dominant groups.

Burnham (2005) also contested "self-reflexivity" as an internal activity and proposed "relational reflexivity" to include others in the reflexive process: children, families, colleagues; indeed, the whole system. Relational reflexivity is an intention, a desire, a process, and a practice of reciprocal engagement and coordination of resources, where professionals and clients co-create a space to explore and experiment with their relationship (Burnham, 2005). An example of relational reflexivity is "questioning the question": Burnham's approach was to ask the family if a certain question was correct, and propose different formulations for them to choose; his intent was to create a reflexive space and trigger family members' reflexivity (as in Ferguson, 2003). This opens new possibilities, new words and languages to name processes or actions, using imagination. By questioning how to go on in a specific situation, the professional is "recalibrating" their communications and actions with the other's help.

In accordance with Burnham (2005) and Daniel (2012), clients need to feel that professionals are sensitive to their responses and that there is some freedom to engage in ways that make sense to them in terms of family and cultural values, scripts, style, myths, and so on. Empowerment and participation are key principles of child protection (Arbeiter \& Toros, 2017; Fuller \& Zhang, 2017; Jackson et al., 2020; Lehtme \& Toros, 2020; Platt, 2012; Toros et al., 2018).

Systemic reflexivity is a relational practice (Munro, 2011; Ruch, 2012; Wonnacott, 2014) that comprises emotions and intuitive knowledge. Jude (2018) referred to interpersonal neuroscience to balance logic and abstract thinking with the contribution of senses, nonverbal signals, and embodied knowledge. Bateson (1972) proposed the term systemic wisdom for the capacity to combine rationality (conscious purpose) with stories, art, play, humor, and the "reasons of the heart" in order to not destroy the delicate balance of living. Systemic reflexivity, then, encourages an active form of research based on holistic attention and "presence", connecting the mind and the body, the conscious and the unconscious, with the relational ongoing context (Formenti, 2017; Jude, 2018). 
International Journal of Child, Youth and Family Studies (2020) 11(4.2): 115-139

As a consequence, following procedures in a linear way and praising reasoning that is based on facts and objective, neutral knowledge may be dangerous and irresponsible in social work, where delicate balances are already jeopardized, and sometimes we cannot even "give proper names to what we are dealing with" (Shotter, 2014, p. 99). Respect for mystery and complexity has to be integrated into social practice and educational care: there are no universally right or wrong answers, or procedures that always work regardless of context.

\section{Reflexivity and Narrativity: The Power of Stories}

So far, this literature review has highlighted the deep connections between narratives and reflexivity. Although we did not find an explicit emphasis on stories, a narrative approach has been proposed as a tool for intervention, a methodology, or an epistemic stance by many authors.

Language and communication are considered to be key tools with which to question ourselves and our practices, and to imagine alternative ideas and actions (McNamee, 2015). Watson (2019) combined systemic ideas with conversation analysis to show the role of talk in fostering change. Language is a determinant resource for therapy and social work: a word can shape or redirect a whole conversation, and systemic techniques offer suggestions for how a conversation can be structured in order to open possibilities; for example, by using certain kinds of questions.

White (1997) developed a hermeneutic approach to social work, based on the narrative therapeutic approach (Epston \& White, 1992), by which people's accounts of their problems are treated as stories that can be listened to and reedited, recognizing in them the influence of hidden and "taken for granted" assumptions. Change can happen when these assumptions are revised through narrative means, revealing that many problematic situations are socially constructed and reproduced through language.

Abduction (metaphors, literary and artistic tools, nonverbal communication) overturns the dominant idea that "simple stories" or "lay accounts" are inferior to professional constructions. Characterized as "thinking in stories" by Bateson (1979), abduction is a process of knowledge that militates against destructive linear interventions. What kinds of stories are built when working with families and children? White (1997) uses Bourdieu's epistemic reflexivity to show how a situation can be assessed, under the interpretative paradigm, in a way that fits lay accounts. For example, interpreting a parent's request for daycare as a form of manipulation is inappropriate. Social workers should abandon a naive realist epistemology based on a linear correspondence between reality and its description. Reality is storied.

The narrative approach suggests that a given situation has multiple interpretations. So, the professionals' task is to search for a more hopeful, more helpful, story (Pocock, 1995). Helpful stories change from case to case and follow the situation as it evolves in time, so the process of helping a client cannot be reduced to recipes. As an example, a child's challenging behavior might be defined as a response to anger over her parents' separation, which is more useful than considering her to be "bad", misbehaving, or even mentally ill. Here, the story is moved from the 
child to the family system. However, a baby's persistent crying may be more usefully narrated as resulting from colic than as a demonstration of the mother's inability (Pocock, 1995). Looking for more helpful stories does not hinder the use of established scientific theories and models (attachment theory, trauma theory, etc.), but it reduces the damage caused by blaming parents for their children's difficulties, which can create guilt and hopelessness.

Some narratives create prisons and are especially disempowering for parents who are unaware of their personal strengths and convinced that they are useless, unlovable, or guilty (Pocock, 1995). Settling on a better story for one person, however, could make things worse for another, perhaps leaving them more vulnerable. Alleviating a mother's guilt must not be allowed to result in harm to the child. Therefore, social workers need to avoid both naive objectivism and anarchic relativism when using stories to nurture creativity, imagination, and hope in open conversations. Reflexivity helps them to analyze their knowledge claims and practices (Taylor \& White, 2001): What do I (or we) really know? And how? Professional knowledge needs to be scrutinized in each situation and never taken for granted. Although Taylor and White (2001) do not mention the narrative method, they do present stories in the form of interviews, letters, and case studies.

Narratives can be used to assist professional growth. We have already mentioned Roets et al. (2017), whose dialogical storytelling approach to report writing increases the observer's awareness of her or his own contribution to the interpretation of a situation.

Another narrative method of enhancing reflexivity is the model of systemic supervision proposed by Partridge (2019): here, the case presenter is seen as a storyteller offering genograms (enhanced family trees) and stories to the supervision group. This technique works best when the presenter is specific about his or her dilemma and provides sufficient detail in telling and contextualizing the story.

Kearns and McArdle (2012) used narrative inquiry (reflexive dyadic interviews) to explore the construction of identity and resilience in newly qualified social workers based in statutory child welfare services. Their stories of transition from university to the workplace show how newcomers learn to make sense of their experiences. Here again, the focus is on the interpretative process, not on workers' lives as such; these stories highlight the narrators' struggles to position themselves in a new social role within the organization and the larger system.

For Kearns and McArdle (2012), reflexivity is understood as the researcher's position in interpreting and communicating: the reflexive dyadic interview is a conversation between peers, rather than just an exchange of questions and answers. Participants identified their own reflexivity as a growing capacity to recognize their personal investment in the job and manage its emotional demands. For example, understanding and naming emotional reactions to challenging situations was thought of as a resource, especially in the beginning of the practice. One aim of this kind of research is to push readers to open their minds and reimagine their practice, reinterpreting their lived experience. In this way, narrative research can become an educational tool. 
Kondrat (1999) encourages practitioners to scrutinize their current narratives - the stories they tell about themselves in a specific situation - using situational self-awareness to be explicit about their narratives and metaphors to themselves and others, revealing how experience is coconstituted in each situation and worlds of meaning are co-created.

Also the clients' reflexive process can be supported by narratives; according to Ferguson (2003), the most vulnerable members of society can become reflexive citizens if they experience a safe relationship with practitioners and welfare agencies, leading to active participation in their own life planning and to them reediting some key aspects of their narratives.

Bingle and Middleton (2019) used stories in relation to systemic hypothesizing: by evoking and composing different versions of reality and resisting dominant discourse and metaphors, it is possible to recognize that some clients' stories are "subjugated" by professional intervention and by the power of context. New metaphors can disrupt normative structures and unhelpful stories. Participation in the multiverse - the reality where many and conflicting stories coexist — can be transformative; the role of social workers is not to establish the truth, or to give instructions, but to be present, alongside parents and children, and provide a space for the co-creation of new stories, meanings, and actions.

\section{Discussion}

The literature review highlighted some features of reflexivity in social work and child protection, and how reflexivity differs from reflection: while reflection is cognitive, individual, and mainly devoted to improving practices, reflexivity aims at creating new meanings and possibilities in a complex system. Reflection is based on reasoned adaptation of actions to desired outcomes; it takes into account meaning and values, and a critical appraisal of the process. In contrast, reflexivity is based on calibration of action (Bateson, 1979) within a changing context or relationship. It is a compositional (Formenti, 2017), second-order, competence: individual and collective; epistemic (i.e., revealing hidden assumptions) as well as embodied and emotional; and connecting different but interdependent levels (micro, meso, and macro), aspects (theory, knowledge, practice, research, learning, languages, and narratives), and dimensions (conscious and unconscious). We call it "systemic reflexivity", in part because the systemic approach is adopted by most papers using the concept of reflexivity.

Reflexivity is a professional posture, attitude, and competence that encompasses reflection; there is a need, in fact, to nurture individual self-awareness and critical thinking, along with the capacity to integrate one's cognitions, emotions, and actions, knowing that they are shaped by the practitioner's biography and by values and assumptions acquired through participation in previous learning contexts. However, the influence of the meso and macro levels contributes to reflexive competence. At the meso level, the quality of teamwork, specific organizational measures and methods, the creation of informal and formal spaces for dialogue, and supervision and case work help the team of co-workers to clarify and challenge the assumptions, theories, and metaphors that 
shape their workplace and practice. Relationships are constitutive of reflexivity since the Other is needed if one is to challenge previous perspectives. These relational spaces have delicate balances: they must be safe, open, and sustained by dialogue and reciprocal engagement.

Reflexivity also enables a focus on power issues that arise between professionals, and with families and children; roles are built through interaction and affected by interpretations. Dominant discourses and structures must be reflexively deconstructed to open possibilities. Reflexivity enables social workers to take a researcher's position in their practice and to bring ethical and political awareness to it.

To sum up, systemic reflexivity is a comprehensive (Krause, 2012) meta-competence with several facets:

- It encompasses recursivity in the process of interpretation.

- It encourages self-reflexivity of both professionals and clients.

- It fosters the critical analysis of multiple contexts and their hidden assumptions.

- It sustains transformation in intervention and in research.

\section{Reflexivity, Feedback, and Calibration}

As noted above, while reflection is mainly devoted to improving practices by adaptation, reflexivity is related to calibration of action (Bateson, 1979): both entail circularity of experience and meaning, of action and thought; they are based on self-corrective sequences of action, effect, and feedback. Feedback is fundamental to any professional practice since action is related to desired outcomes. Adaptation happens when error is identified and corrected. Calibration comes into play when direct feedback is not enough. In education and care settings, only rarely can errors be detected and corrected immediately; more often, we must compile and interpret information from different senses and sources. In many cases, it is not the single action that needs to be assessed or modified, but the category of actions, the context, and the relationship. With calibration,

to achieve any improvement, correction must be performed upon a large class of actions ... By long practice, [the professional] must adjust the setting of his nerves and muscles so that in the critical event, he will "automatically" give an optimum performance. (Bateson, 1979, p. 2016)

Competence needs calibration. It is built over time, combining multiple streams of information from outside and inside, in different contexts where similar actions are interpreted as belonging to the same class. Applying action correctly or achieving a result does not of itself indicate a competent practitioner. A pedagogical frame based on competence does not define learning as primarily the cumulative acquisition of knowledge and skills. Rather, learning is seen as the capacity for adaptation and calibration within a particular context, using both familiar and new sources of information in a skilled and deliberate way to not only achieve a desired result but to redefine it if necessary. Competence brings interpretation into the unconscious: while newcomers 
need to be aware of what they do, step by step, experts can take rapid decisions by intuition even before their rationality is called into action. Intentionality, however, is always present in the background: competent professionals accept responsibility for continuously assessing their own actions at some level.

Competences from earlier learning and previously handled situations and tasks are integrated in new experiences; human beings tend to use what has already worked in other circumstances, generalizing and transferring it to new situations. Without a re-examination of previous experience and the resulting learning, a professional may forget how a specific competence was acquired; once this is done, however, he or she can connect it to a present situation, and eventually transform it for application to future situations. This meta-competence is thus a basis for opening new possibilities.

\section{Towards a Learning Module on Reflexivity}

Within the ERCCI project, we developed a module on reflexivity for the MOOC. The module is designed to be used in a self-directed way by new and experienced professionals in the workplace. It combines Mezirow's (1991) theory of transformative learning with a systemic and narrative approach; it implements the idea of constructing stories about complex situations in RCC and then using reflexivity in several ways: to search for hidden assumptions in the stories (and in the learner), to connect different sources of knowledge, and to develop a critical interpretation and position at each level: micro, meso, and macro.

To develop the module, we gathered stories from interviews, focus groups, and participatory workshops attended by some 50 experienced professionals, who were asked to identify competences and learning needs in RCC, and give examples related to them. Their varied stories are emotionally and cognitively engaging; they tell of successful and unsuccessful actions; situations whose meaning is not clear; and errors, accidents, and uncertainty; as well as positive achievements. Each story was processed by a group of 10 practitioners who were also students in pedagogical sciences and collaborators of the project: they explored together different meanings entailed by the stories, using writing, abduction, and dialogue in order to multiply and clarify interpretations.

For each story, they used questions as interpretative guidelines to encourage reflection, reflexivity, and a research orientation:

- What happened in the situation? How would you represent it? How would you behave in similar circumstances? What can you learn from this story, as told? (reflection)

- How many points of view and interpretations emerged? Do these reveal assumptions at a micro, meso, and macro level? Are there conflicts, dilemmas, or alternative views? What can be learnt by integrating all these interpretations? (reflexivity) 
International Journal of Child, Youth and Family Studies (2020) 11(4.2): 115-139

- Are there other possible narratives? How would you search for new versions of the situation? What would you need to know, to do, or to learn in order to open more possibilities? (research)

This process brought us to a long list of competences, from which we have chosen five reflexive competences that we intend to focus on and help students to develop through our learning module (composed of five units): (a) responsivity; (b) reciprocity in relationships; (c) creative conflict management; (d) multiplying descriptions and making systemic hypotheses; and (e) developing the cycle of intervention. A short presentation of each competence in relation to our definition of reflexivity as the meta-competence follows.

\section{Responsivity}

A competent RCC worker is sensitive to feedback (Burnham, 2005; Jude, 2018) and able to communicate effectively in an emergent situation. It can be defined as emotional presence (Krueger, 1994), openness, attunement, attention, embodied action, context reading, and interpersonal intelligence. It entails a capacity of being, doing, and interpreting "with" (Garfat et al., 2018) children, families, and other professionals. It goes beyond listening or empathy, as it recognizes circularity and interdependence, so that practitioners take responsibility for their contributions in each situation. It is also related to rhythm in being a shared experience of engagement in a dynamic interconnection (Krueger, 1994; Maier, 1992) that respects different temporalities, identities, and expectations. The reflexive focus is on the ongoing interaction, movements, and feedback, nurtured by emotional, cognitive, and epistemic self-awareness.

RCC professionals are constantly called to join in the flow of multiple actions. Responsivity helps them to recognize their present position, state of mind, and feelings in relation to others and to take responsibility for the climate in the workplace and the effects of their choices, without claiming a false neutrality or, on the other side, control of the situation.

\section{Reciprocity in relationships}

Reflexivity entails relationality (Burnham, 2005; Ferguson, 2003; Jude, 2018) — the awareness of interdependence in human relationships. Positions are not neutral: they are shaped by gender, class, culture, and previous experience. Formenti (2017) identifies four relational postures adopted by learners in educational care:

- Awaiting — accepting being taught, guided, and assessed by someone who "knows".

- Taking refuge - complying by doing what is needed, asked, and planned in order to achieve an agreed result.

- Being intentional — bringing one's interests, desires, or needs; asking to be listened to.

- Giving up control - being curious, open, and willing to play on a peer-to-peer basis. 
Each of these positions also depends on the other's positioning: there is a learner-educator complementarity between awaiting and teaching, taking refuge and coaching, being intentional and listening, giving up control and reciprocating. "Relational reflexivity" (Burnham, 2005; Jude, 2018) allows us to name our own positioning and that of the other, and to sustain the evolution of the relationship. Self-reflexiveness (Why do I take this position? How did I learn it? How can I develop a richer relational repertoire? And what about the other?), as well as shared and cultural reflexiveness, help move the relationship to a new stance. In this view, there is no good or bad position: what is interesting is our capacity to change and find what is helpful in the situation. For example, the position of awaiting entails being able to receive, stay silent, be humble, give power to the other, and accept that the other knows more. It may be used to confirm power, but also to reverse the power dynamic. For a worker in RCC, awaiting directions from management may feel right, comfortable, and sometimes even helpful, but may also be irresponsible on many occasions.

Taking refuge entails being guided by expert knowledge. An expert knows the situation, context, rules, and tasks; the expert's role is to prescribe attitudes and behaviors, encouraging the client to do what is needed. In RCC, a newcomer may take the posture of refuge towards an experienced colleague; however, this could make it harder to challenge hidden assumptions.

Educational care welcomes intentionality as the expression of self-knowledge and personal responsibility. In RCC, it is a desirable outcome for everybody: for the children, to achieve autonomy in the long run; for parents, to nurture responsible parenting; and for professionals, to sustain deliberate action. It may become a problem when intentionality does not consider other points of view, constraints, or organizational rules. Here again, reflexivity helps to enact the multiverse and sustain shared, system-wise, decisions.

Giving up control is a playful posture that happens when we are relaxed, trusting, and able to leave our comfort zone. In RCC, learning from each other is always a possibility, but learning may be precluded by discourses of control, child protection and safety, roles, power, and family blaming. Abduction (Bateson, 1979; Pocock, 1995; White, 1997) — using play, art, body language, metaphors, and stories - helps to smooth defensiveness and ease rigidity, sustaining the practitioners' capacity to interact playfully, to get involved, and to take on challenges. Reflexivity sustains playfulness when controlling talk and defences can be named, shared, and transformed.

\section{Creative conflict: Emotional self-awareness, active listening, and humor}

Creative conflict management (Sclavi, 2008) requires the capacity to welcome conflict - not avoiding or denying it but taking it as an opportunity to transform our frames of meaning. Active listening, emotional self-awareness, and humor help one recognize the cultural frameworks of meaning that produce conflict. We learn from each other if we adopt a dialogic method, nurturing humor (i.e., a distancing from our assumptions) as a cognitive, emotional, and epistemic competence. In RCC, playfulness and humor are very relevant; they bring lightness and a livable climate, but they need intelligence and attunement to balance between, on the one hand, providing 
a contrast to heaviness, worries, moralism, and negative thinking and, on the other hand, avoiding superficiality or underestimation of personal struggles. "Thick active listening" is different from empathic listening. It recognizes complexity and the multiverse: the same situation has different meanings for different actors. In RCC, empathy is highly desirable, but also a risk: the observer never escapes his or her framework, and thus must never take for granted being able to "really understand" the other.

\section{Multiplying descriptions and making systemic hypotheses}

A main feature of systemic reflexivity is curiosity (Bingle \& Middleton, 2019; Dugmore et al., 2018; Partridge et al., 2019): a story may be permeated by a single narrow viewpoint or structured by dominant views, so the capacity to listen to multiple voices and combine different sources of knowledge (Jude, 2018) brings recognition that any story presents just one version of reality. Multiplying stories is a way to enact the multiverse (Bingle \& Middleton, 2019), allowing critical analysis (Fook, 2012), cultural reflexivity (Daniel, 2012), and transformation.

Hypothesizing helps RCC workers to distance themselves from naive interpretations of a situation and open up possibilities. Abduction is also a way to multiply stories (Bateson, 1979; Pocock, 1995; White, 1997). In RCC, there may be silenced stories and voices; there is a high risk of building stereotypical stories (e.g., taking for granted that parents have not properly cared for their children). Teamwork is a good setting in which to elaborate systemic hypotheses, bringing different perspectives to the workplace; however, this needs time, a methodical approach, and specific organizational measures.

\section{Developing the cycle of intervention}

Reflexivity invites professionals to become researchers of their own practice (Fook, 2012; Jude, 2018; Shotter, 2016) rather than allow bureaucracy and managerialism to limit their critical thinking. Good child care intervention is planned, monitored, and evaluated as part of an ongoing assessment of the situation. Individualized educational planning, a common tool of intervention, can be empowered by a participatory reflexive approach, connecting different data and narratives within systemic hypotheses and involving all the actors (children, families, RCC workers, other professionals, and agencies). The reflexive approach — between professionals and with clients shapes all the phases of intervention, including monitoring and evaluation. Intervention is described as complex and recursive, not as the mere delivery of a service.

In sum, these five competences (the learning module's units) will sustain learners in questioning professional action and interaction; they will nurture complex understanding and the capacity to use reflection, reflexivity, and research when acting within complex relational environments. The next phase (from January to June 2021) will be the implementation and testing of the module, by which we expect to collect evidence that learning these reflexive competences empowers professional growth and awareness in RCC. The final version of the MOOC should be available online in October 2021. 
International Journal of Child, Youth and Family Studies (2020) 11(4.2): 115-139

\section{Conclusion}

The complex situations that RCC workers deal with are often overwhelming and disorientating. They are called daily to act deliberately and ethically, with intelligence, heart, imagination, and a pragmatic attitude; and they are required to take timely decisions, solve unexpected problems, and transform conflicts and crises into learning opportunities. A "good-hearted" profile does not on its own guarantee the best experience to children and their families. In child protection, many struggles and conflicts concern rival interpretations of what is going on or what should be done. The desire to achieve an ultimate truth and "do the right thing" haunts the learning experience and professional growth of newcomers. However, relationships are inherently subject to change and uncertainty (Rothuizen \& Harbo, 2017); there are no recipes to manage this complexity. Reflexivity is a way to learn by complexity, when it is seen as a constitutive feature of life and of human relationships, and not as a problem.

Educational care is the work of a team. The systemic approach is critical of the dominant individualistic view of education, which focuses on the one-to-one relationship between an adult and a child. Life in a residential unit is a collective experience that needs coordination. Groups and subgroups play a fundamental role; conflicts, alliances, and coalitions are common. Practitioners must coordinate their actions with co-workers and managers, administrators, families, and other professionals and agencies. A pedagogically explicit model based on reflexivity can sustain coordination.

In addition, reflexivity allows one to compose different models and overcome binary thinking and the clash of paradigms. While the relational perspective is still dominant in RCC, professionalism, specialization, and evidence-based practices are increasing, as well as bureaucratization and standards. A systemic and critical approach, calling for more participation of children and families, and bringing to the forefront social justice, personalization of treatment, and respect for cultural differences, is also valued. In this evolving frame, a team of reflexive professionals is empowered by their capacity for calibrating their actions in relation to specific situations; taking overt positions; recognizing circularity and interdependence in relationships with children, parents, and colleagues; welcoming conflicts as opportunities; and using emotional selfawareness, thick active listening, and humor to sustain transformation. They will also be able to use stories, play, and aesthetic languages to multiply descriptions and celebrate differences, develop reciprocal trust, and overcome ideological truths, while striving to bring about desirable outcomes for all concerned.

We defined reflexivity as a second-order competence that entails a capacity of calibration of action, enacted within a context, sensitive to meaning, and open to the new. We proposed using reflexivity to overcome individualism and integrate the group (meso) and culture (macro) in the thinking that orients practice. Competence is transferrable: what is learned reflexively can be used in other contexts. Last but not least, this model of reflexivity is contagious: when practitioners become competent in it, it becomes natural for them to propose the same to children and families, offering innovative spaces for voice and transformation. 
International Journal of Child, Youth and Family Studies (2020) 11(4.2): 115-139

\section{References}

Akin, B. A., Brook, J., Byers, K. D., \& Lloyd, M. H. (2016). Worker perspectives from the front line: Implementation of evidence-based interventions in child welfare settings. Journal of Child and Family Studies, 25, 870-882. doi:10.1007/s10826-015-0283-7

Alvesson, M., \& Sköldberg, K. (2000). Reflexive methodology. Sage.

Andrew, Y. (2015). I'm strong within myself: Gender, class and emotional capital in childcare. British Journal of Sociology of Education, 36(5), 651-668. doi:10.1080/01425692.2013.835711

Arbeiter, E., \& Toros, K. (2017). Participatory discourse: Engagement in the context of child protection assessment practices from the perspectives of child protection workers, parents and children. Children and Youth Services Review, 74, 17-27. doi:10.1016/i.childyouth.2017.01.020

Ball, K. (2013). Action learning: Creating a space for multi-agency reflexivity to complement case management. Practice Social Work in Action, 25(5), 335-347. $\underline{\text { doi:10.1080/09503153.2013.860090 }}$

Bateson, G. (1972). Steps to an ecology of mind: Collected essays in anthropology, psychiatry, evolution, and epistemology. Ballantine.

Bateson, G. (1979). Mind and nature: A necessary unity. Ballantine.

Bingle, L., \& Middleton, A. (2019). From doing to being: The tensions of systemic practice in social work - group reflective supervision in child protection. Journal of Family Therapy, 41(3), 384-406. doi:10.1111/1467-6427.12256

Burnham, J. (2005). Relational reflexivity: A tool for socially constructing therapeutic relationships. In C. Flaskas, B. Mason, \& A. Perlesz (Eds.), The space between: Experience, context and process in the therapeutic relationships (pp. 1-19). Karnac.

Cameron, C., Elliott, H., Iqbal, H., Munro, E., \& Owen, C. (2016). Focus on practice in three London boroughs: An evaluation.

https://assets.publishing.service.gov.uk/government/uploads/system/uploads/attachment_data/ file/534394/Triborough_focus_on_practice_July_2016.pdf

Cecchin, G. (1987). Hypothesising, circularity, and neutrality revisited: An invitation to curiosity. Family Process, 26(4), 405-413. doi:10.1111/j.1545-5300.1987.00405.x

Cronen, V. E., Johnson, K. M., \& Lannamann, J. W. (1982). Paradoxes, double binds, and reflexive loops: An alternative theoretical perspective. Family Process, 21, 91-112. doi:10.1111/j.1545-5300.1982.00091.x 
International Journal of Child, Youth and Family Studies (2020) 11(4.2): 115-139

D’Cruz, H., Gillingham, P., \& Melendez, S. (2007). Reflexivity, its meanings and relevance for social work: A critical review of the literature. British Journal of Social Work, 37, 73-90. $\underline{\text { doi:10.1093/bjsw/bcl001 }}$

Daniel, G. (2012). With an exile's eye: Developing positions of cultural reflexivity (with a bit of help from feminism). In I.-B. Krause (Ed.), Culture and reflexivity in systemic psychotherapy: Mutual perspectives (pp. 91-113). Routledge.

del Valle, J. F., Sainero, A. M, \& Bravo, A. (2015). Needs and characteristics of high-resource using children and youth. In J. K. Whittaker, J. F. del Valle, \& L. Holmes (Eds.), Therapeutic residential care for children and youth: Developing evidence-based international practice (pp. 49-61). Jessica Kingsley.

Dugmore, P., Partridge, K., Sethi, I., \& Krupa-Flasinska, M. (2018). Systemic supervision in statutory social work in the UK: Systemic rucksacks and bells that ring. European Journal of Social Work, 21(3), 400-414. doi:10.1080/13691457.2018.1446914

Epston, D., \& White, M. (1992). Experience and contradiction, narrative and imagination. Dulwich Centre.

Ferguson, H. (1997). Protecting children in new times: Child protection and the risk society. Child \& Family Social Work, 2(4), 221-234. doi:10.1046/j.1365-2206.1997.00064.x

Ferguson, H. (2003). Welfare, social exclusion and reflexivity: The case of child and woman protection. Journal of Social Policy, 32(2), 199-216. doi:10.1017/S0047279402006967

Ferguson, H. (2018). How social workers reflect in action and when and why they don't: The possibilities and limits to reflective practice in social work. Social Work Education, 37(4), 415-427. doi:10.1080/02615479.2017.1413083

Fook, J. (2002). Social work: Critical theory and practice. Sage.

Fook, J. (2012). The challenges of creating critically reflective groups. Social Work With Groups, 35(3), 218-234. doi:10.1080/01609513.2011.624375

Formenti, L. (2017). Formazione e trasformazione. Un modello complesso [Training and transformation: A complex model]. Raffaello Cortina.

Formenti, L., Galimberti, A., \& Ferrari, M. (2020). Care leavers in Italy: From 'vulnerable' children to 'autonomous' adults? In H. Wright \& M. Hoyen (Eds.), Discourses we live by: Narratives of educational and social endeavour (pp. 245-268). Open Book.

Formenti, L., \& West, L. (2018). Transforming perspectives in lifelong learning and adult education: A dialogue. Palgrave Macmillan. 
International Journal of Child, Youth and Family Studies (2020) 11(4.2): 115-139

Forrester, D. (2016). What, when, why and how: Zombie social work and the need for a new narrative. In E. Solomon (Ed.), Rethinking children's services fit for the future? (pp. 8-13). National Children's Bureau. Retrieved from https://www.ncb.org.uk/about-us/rethinkingchildrens-services

Fuller, T., \& Zhang, S. (2017). The impact of family engagement and child welfare services on maltreatment re-reports and substantiated re-reports. Child Maltreatment, 22(3), 183-193. $\underline{\text { doi:10.1177/1077559517709996 }}$

Garfat, T. (2013). The meaningful use of everyday life events in child and youth. In T. Garfat, L. Fulcher, \& J. Digney (Eds), Making moments meaningful in child \& youth care practice (pp. 1-6). The CYC-Net Press.

Garfat, T., Freeman, J. Gharabaghi, K., \& Fulcher, L. (2018). Characteristics of a relational child and youth care approach revisited. E-journal of the International Child and Youth Care Network (CYC-Net), 236, 7-44. https://www.cyc-net.org/cyc-online/oct2018.pdf

Gharabaghi, K. (2020). Being a good child and youth care practitioner. E-journal of the International Child and Youth Care Network (CYC-Net), 252, 10-13. https://www.cycnet.org/cyc-online/feb2020.pdf

Goodman, S., \& Trowler, I. (2012). Social work reclaimed: Innovative frameworks for child and family social work practice. Jessica Kingsley.

Harder, T., Knorth, E. J., \& Kalverboer, M. E. (2017). The inside out? Views of young people, parents and professionals regarding successful secure residential care. Child and Adolescent Social Work Journal, 34, 431-441. doi:10.1007/s10560-016-0473-1

James, S. (2017). Implementing evidence-based practice in residential care: How far have we come? Residential Treatment for Children and Youth, 34(2), 155-175. doi:10.1080/0886571X.2017.1332330

Jackson, S., Kelly, L., \& Lesli, B. (2020). Parental participation in child protection case conferences. Child \& Family Social Work, 25, 421-429. doi:10.1111/cfs.12698

Jude, J. (2018). The practice of systemic reflexivity. Journal of Social Work Practice, 32(1), 4557. doi:10.1080/02650533.2017.1291499

Kearns, S., \& McArdle, K. (2012). Doing it right? Accessing the narratives of identity of newly qualified social workers through the lens of resilience: 'I am, I have, I can'. Child \& Family Social Work, 17(4), 385-394. doi:10.1111/j.1365-2206.2011.00792.x

Knorth, E. J., Harder, A. T., Huyghen, A. M. N., Kalverboer, M. E., \& Zandberg, T. (2010). Residential youth care and treatment research: Care workers as key factor in outcomes? International Journal of Child and Family Welfare, 13(1-2), 49-67. 
International Journal of Child, Youth and Family Studies (2020) 11(4.2): 115-139

Kondrat, M. E. (1999). Who is the 'self' in self-aware: Professional self-awareness from a critical theory perspective. Social Service Review (December 1999), 451-477. $\underline{\text { doi:10.1086/514441 }}$

Krause, I.-B. (2012). Culture and the reflexive subject in systemic psychotherapy. In I.-B. Krause (Ed.), Culture and reflexivity in systemic psychotherapy: Mutual perspectives (pp. 1-36). Routledge.

Krueger, M. (1994). Framing child and youth care in moments of rhythm, presence, meaning, and atmosphere. Child \& Youth Care Forum, 23(4), 223-229. doi:10.1007/BF02209086

Lehtme, R., \& Toros, K. (2020). Parental engagement in child protection assessment practice: Voices from parents. Children and Youth Services Review, 113, 104968. doi:10.1016/j.childyouth.2020.104968

Magnuson, D., Patten, N., \& Looysen, K. (2012). Negotiation as a style in child protection work. Child \& Family Social Work, 17(3), 296-305. doi:10.1111/j.1365-2206.2011.00780.x

Maier, H. W. (1992). Rhythmicity - A powerful force for experiencing unity and personal connections. Journal of Child and Youth Care Work, 8, 7-13. https://www.cyc-net.org/cyconline/cycol-0704-rhythmicity.html

Maton, K. (2003). Reflexivity, relationism, \& research: Pierre Bourdieu and the epistemic conditions of social scientific knowledge. Space and Culture, 6(1), 52-65. doi:10.1177/1206331202238962

Mattingly, M., Stuart, C., \& VanderVen, K. (2012). Competencies for professional child and youth work practitioners: An overview. Journal of Child and Youth Care Work, 24, 16-24.

McNamee, S. (2009). Postmodern psychotherapeutic ethics: Relational responsibility in practice. Human Systems: The Journal of Therapy, Consultation \& Training, 20(1), 57-71.

McNamee, S. (2015). Ethics as discursive potential. Australian and New Zealand Journal of Family Therapy, 36, 419-433. doi:10.1002/anzf.1125

Mezirow, J. (1991). Transformative dimensions of adult learning. Jossey-Bass.

Monson, T. A. (2020). Risk attitudes within 'complex youth' assessment and decision making: Professional perspectives. Child Care in Practice, 26(2), 210-222. doi:10.1080/13575279.2019.1685462

Munro, E. (2011). The Munro review of child protection: Final report. A child-centred system. https://assets.publishing.service.gov.uk/government/uploads/system/uploads/attachment_data/ file/175391/Munro-Review.pdf 
International Journal of Child, Youth and Family Studies (2020) 11(4.2): 115-139

Papp, E., \& Rácz, A. (2016). How professionals in the social system and child protection perceive their profession. Acta Universitatis Sapientiae - Social Analysis, 6(1), 71-91.

Partridge, K., Dugmore, P., Mahaffey, H., Chidgey, M., \& Owen, J. (2019). 'Step by step, side by side': The quest to create relational artistry through systemic practice within children's social care. Journal of Family Therapy, 41(3), 321-342. doi:10.1111/1467-6427.12262h

Pearce, W. B., \& Cronen, V. E. (1980). Communication, action and meaning. Praeger.

Platt, D. (2012). Understanding parental engagement with child welfare services: An integrated model. Child and Family Social Work, 17(2), 138-148. doi:10.1111/j.1365$\underline{2206.2012 .00828 . x}$

Pocock, D. (1995). Searching for a better story: Harnessing modern and postmodern positions in family therapy. Journal of Family Therapy, 17, 149-173. doi:10.1111/j.14676427.1995.tb00011.x

Roets, G., Roose, R., De Wilde, L., \& Vanobbergen, B. (2017). Framing the 'child at risk' in social work reports: Truth-telling or storytelling? Journal of Social Work, 17(4), 453-469. $\underline{\text { doi:10.1177/1468017316644864 }}$

Rothuizen, J. J., \& Harbo, L. J. (2017). Social pedagogy: An approach without fixed recipes. International Journal of Social Pedagogy, 6(1), 6-28. doi:10.14324/111.444.ijsp.2017.v6.1.002

Ruch, G. (2012). Where have all the feelings gone? Developing reflective and relationship-based management in childcare social work. British Journal of Social Work, 42(7), 1315-1332. doi:10.1093/bjsw/bcr134

Santello, F., Colombini, S., Ius, M., \& Milani, P. (2017). P.I.P.P.I.: What has changed? How and why? The empirical evidence. Rivista Italiana di Educazione Familiare, 2, 111-136. doi:10.13128/RIEF-22396

Schön, D. (1983). The reflective practitioner: How professionals think in action. Basic.

Sclavi, M. (2008). The role of play and humor in creative conflict management. Negotiation Journal, 24(2), 157-180.

Sebba, J., Luke, N., McNeish, D., \& Rees, A. (2017). Children's Social Care Innovation Programme: Final evaluation report. https://innovationcsc.co.uk/wpcontent/uploads/2017/11/CSCIP_Final_evaluation_report.pdf

Selvini Palazzoli, M., Boscolo, L., Cecchin, G., \& Prata, G. (1980). Hypothesizing - circularity — neutrality: Three guidelines for the conductor of the session. Family Process, 19(1), 3-12. doi:10.1111/j.1545-5300.1980.00003.x 
International Journal of Child, Youth and Family Studies (2020) 11(4.2): 115-139

Shotter, J. (2014). Getting it: Withness-thinking and the dialogical... in practice. Hampton.

Shotter, J. (2016). Speaking, actually: Towards a new 'fluid' common-sense understanding of relational becomings. Everything is Connected.

Simon, G., \& Chard, A. (2014). Systemic inquiry: Innovations in reflexive practice research. Everything is Connected.

Taylor, C., \& White, S. (2001). Knowledge, truth and reflexivity: The problem of judgement in social work. Journal of Social Work, 1(1), 37-59. doi:10.1177/146801730100100104

Timonen-Kallio, E., \& Hämäläinen, J. (2019). Social pedagogy-informed residential child care. International Journal of Social Pedagogy, 7(1), 1-14.

doi:10.14324/111.444.ijsp.2019.v7.1.010

Toros, K., DiNitto, D. M., \& Tiko, A. (2018). Family engagement in the child welfare system: A scoping review. Children and Youth Services Review, 88, 598-607. doi:10.1016/j.childyouth.2018.03.011

von Foerster, H. (2003). Understanding understanding: Essays on cybernetics and cognition. Springer.

Watson, R. (2019). Jointly created authority: A conversation analysis of how power is managed by parents and systemic psychotherapists in children's social care. Journal of Family Therapy, 4(3), 357-383. doi:10.1111/1467-6427.12244

White, S. (1997). Beyond retroduction? Hermeneutics, reflexivity and social work practice. The British Journal of Social Work, 27(5), 739-753.

Wonnacott, J. (2014). Developing and supporting effective staff supervision. Pavilion. 